\title{
New mechanisms of droplet coarsening in phase-separating fluid mixtures
}

\author{
Hajime Tanaka \\ Institute of Industrial Science, University of Tokyo, Minato-ku, Tokyo 106, Japan.
}

(Received 21 October 1996)

\begin{abstract}
We propose here a new mechanism of droplet coarsening in phase-separating fluid mixtures. In contrast to the conventional understanding that there are no interactions between droplets in the late stage of spinodal decomposition, we demonstrate the existence of interactions between droplets that is caused by the coupling between diffuse concentration change around droplets. We show the possibility that this mechanism plays an important role in droplet phase separation together with Brownian-coagulation mechanism. We also discuss the coupling between hydrodynamic and diffusion modes, namely, "collision-induced collision" phenomena.
\end{abstract}

PACS numbers:64.70.-p, 64.75.+g, 05.70.Fh

Droplet patterns are generally observed in the late stage of phase separation in binary mixtures of solids and liquids at off-symmetric compositions [1, 2. There are two well-known coarsening mechanisms for droplet phase separation: (i) evaporation-condensation mechanism [1.33 and (ii) Brownian-coagulation mechanism due to collision between droplets that is caused by the free, thermal diffusion of droplets without any interaction [1., A. 5]. The former is known as Lifshitz-Slyozov-Wagner (LSW) mechanism and the latter as Binder-Stauffer (BS) mechanism. Both mechanisms give the same form of the power law of for the evolution of the mean droplet radius $R$ as a function of the phase-separation time $t$, in three dimensions [7] 10]:

$$
R^{3}=k_{d}\left(k_{\mathrm{B}} T / 5 \pi \eta\right) t
$$

where $k_{d}$ is a constant, $T$ is the temperature, $\eta$ is the viscosity, and $k_{\mathrm{B}}$ is Boltzmann's constant. For LSW mechanism $k_{d}=0.053$, while for BS mechanism $k_{d}=6 \Phi_{d}$ $\left(\Phi_{d}\right.$ : the volume fraction of the minority phase) [5.6. 6 . The droplet coarsening behavior in phase-separating fluid mixtures has mostly been explained in terms of these LSW and BS mechanisms so far. Recently, however, some reserachers start to think that this well-accepted view needs some essential modifications in physics on the basis of experimental results and theoretical considerations. There are two important physical factors that have so far been ignored: (i) interdroplet interactions 11 15 and (ii) hydrodynamic interactions 14, 16].

In this Communication, we consider the interdroplet interactions between droplets and the resulting hydrodynamic motion of droplets [17]. We think that the conventional picture that a droplet moves around freely by Brownian motion and accidentally collides with another droplet is not correct in the exact sense, at least, for a system of a high droplet density 12,13].

The kinetic equations for classical binary fluids are [1]

$$
\frac{\partial \phi}{\partial t}=-\vec{\nabla} \cdot(\phi \vec{v})+L_{0} \nabla^{2} \frac{\delta(\beta H)}{\delta \phi}+\theta
$$

$$
\rho \frac{\partial \vec{v}}{\partial t}=\vec{F}_{\phi}-\nabla p+\eta \nabla^{2} \vec{v}+\vec{\iota}
$$

where $\phi$ is the composition, $\vec{v}$ is the velocity, $\rho$ is the density, $p$ is a part of the pressure that is determined to satisfy the incompressibility condition, $\theta$ and $\vec{\iota}$ are thermal noises, and $\beta=1 / k_{\mathrm{B}} T$. Here $H$ is the GinzburgLandau-type Hamiltonian:

$$
H=k_{\mathrm{B}} T \int d \vec{r}\left[f(\phi)+\frac{K}{2}(\nabla \phi)^{2}\right]
$$

with $f(\phi)=-\frac{\tau}{2} \phi^{2}+\frac{u}{4} \phi^{4}$. In Eq. (3), $\vec{F}_{\phi}$ is the thermodynamic force density acting on the fluid due to the fluctuations of the composition $\phi$ that is given by

$$
\frac{\vec{F}_{\phi}}{k_{\mathrm{B}} T}=-\phi \nabla \mu=-\nabla\left[\pi-K \nabla\left(\phi \nabla^{2} \phi\right)\right]-K \nabla^{2} \phi \nabla \phi,
$$

where $\mu=\delta(\beta H) / \delta \phi$ is the chemical potential and $\pi$ is the osmotic pressure given by $\pi=\phi \partial f / \partial \phi-f$. The conserved part of the above thermodynamic force, $\vec{F}_{\phi}^{c} / k_{\mathrm{B}} T=-\nabla\left[\pi-K \nabla\left(\phi \nabla^{2} \phi\right)\right]$, cannot produce any velocity fields under the incompressibility condition $\vec{\nabla} \cdot \vec{v}=$ 0 and it has to be completely balanced with a part of $\nabla p$. Accordingly, only a part of the other force, $\vec{F}_{\phi}^{i n t}=$ $-K \nabla^{2} \phi \nabla \phi$, can produce the velocity fields through Eq. (3). Using the steady-state approximation $(\partial \vec{v} / \partial t \sim 0)$, thus, we obtain the following expression of $\vec{v}[1,18]$ :

$$
\vec{v}=\int d \overrightarrow{r^{\prime}} \boldsymbol{T}\left(\vec{r}-\overrightarrow{r^{\prime}}\right) \cdot \vec{F}_{\phi}^{i n t}\left(\overrightarrow{r^{\prime}}\right),
$$

where $\boldsymbol{T}$ is the so-called Oseen tensor.

This expression gives us the physical insight on hydrodynamic effects on domain coarsening as follows [2, 19, 20]. After the formation of a sharp interface, it has so far been assumed that the interface profile can be described by $\phi_{\text {int }}=\phi_{e} \tanh (\zeta / \sqrt{2} \xi)$ [21], where $\phi_{e}(=\sqrt{\tau / u})$ is the equilibrium composition, $\xi$ is the correlation length. $\zeta$ is the distance from the interface defined by $\zeta=\vec{n} \cdot\left(\vec{r}-\vec{r}_{i n t}\right)$, where $\vec{r}_{\text {int }}$ is a point on the interface and $\vec{n}$ is the unit 
normal vector at the point $\vec{r}_{\text {int }}$ toward the domain with a positive value of $\phi$. Then, the thermodynamic force density $\vec{F}_{\phi}^{\text {int }}$ can be expressed by

$$
\frac{\vec{F}_{\phi}^{i n t}}{k_{\mathrm{B}} T}=-K(\vec{\nabla} \cdot \vec{n})\left(\frac{\partial \phi_{i n t}}{\partial \zeta}\right)^{2} \vec{n}
$$

Note that $\vec{\nabla} \cdot \vec{n}$ is the curvature at $\vec{r}_{i n t}$ and $\vec{\nabla} \cdot \vec{n}=$ $1 / R_{1}+1 / R_{2}$, where $1 / R_{1}$ and $1 / R_{2}$ are the two principal curvatures of the interface. Here we use the relations:

$$
\begin{aligned}
\nabla \phi & \cong\left(\partial \phi_{i n t} / \partial \zeta\right) \vec{n}, \\
\nabla^{2} \phi & \cong \frac{\partial^{2} \phi_{i n t}}{\partial \zeta^{2}}+\left(\frac{\partial \phi_{i n t}}{\partial \zeta}\right) \vec{\nabla} \cdot \vec{n} .
\end{aligned}
$$

Thus, we obtain the following equation by putting Eq. (6) into Eq. (3) and using $k_{\mathrm{B}} T K\left(\partial \phi_{\text {int }} / \partial \zeta\right)^{2} \cong \sigma \delta(\zeta)$ :

$$
-\nabla p^{\prime}-\sigma\left(\frac{1}{R_{1}}+\frac{1}{R_{2}}\right) \delta(\zeta) \vec{n}+\eta \nabla^{2} \vec{v}=0,
$$

where $\sigma$ is the interface tension. It should be noted that Eq. (9) is dependent on $\Phi_{d}$ through the characteristic curvatures of the interface. Under the incompressibility condition, thus, the domain geometry ( $\vec{n}$ pattern) dominates the coarsening mechanism: For a nearly symmetric composition $\left(\Phi_{d} \sim 1 / 2\right)$, we have a bicontinuous pattern; and, thus, the second term in Eq. (9) produces the velocity fields of $v \sim \sigma / \eta$ that lead to the growth law of $R \sim(\sigma / \eta) t$ (Siggia's mechanism). For an off-symmetric composition $\left(\Phi_{d} \neq 1 / 2\right)$, on the other hand, we have a droplet pattern; and, thus, the second term in Eq. (9) is balanced with $\nabla p^{\prime}$ to satisfy $\nabla \cdot \vec{v}=0$. Thus, there is a pressure difference of $2 \sigma / R$ across the interface (Laplace's law), while there are no macroscopic velocity fields $\vec{v}=0$ except during the coalescence process. The latter fact is the basis of Brownian-coagulation mechanism, which assumes that there is no interparticle interaction and the droplet motion is purely driven by thermal velocity noises [ $\vec{\iota}$ in Eq. (3)].

Here we would like to raise a fundamental question of whether droplets can feel neighboring droplets or not. The above argument tells us that the answer is "no". However, we point out that in the above argument there are deficiencies that can be important particularly in phase separation of off-symmetric binary mixture; namely, (i) the gradual concentration change around a droplet is neglected above and the concentration profile is wrongly assumed to be always described by $\phi_{\text {int }}$ along the direction perpendicular to the interface and (ii) hydrodynamic flow induced by droplet collision is neglected. We focus here mainly on the problem (i). Since the matrix can never be in equilibrium with droplets with different sizes at the same time, a droplet usually has long-range concentration (diffusion) fields around it, as recently pointed out by us 12,14 and Karpov [15]. We think that this fact plays an important role in droplet coarsening, especially for fluid mixtures: The finite curvature of droplets naturally leads to the diffuse concentration change around them and the coupling between these concentration fields causes interactions between droplets. Experimental evidence of such interactions between droplets has recently been reported for fluid mixtures by Aver'yanov and Kalmykov [11] and Tanaka [12].

Before discussing the dynamics, we need to know the quasi-stationary concentration profile around a droplet for estimating the strength of the interdroplet interaction. After the formation of a sharp interface, for both spinodal decomposition (SD) and nucleation and growth (NG), we have $\partial \delta \phi / \partial t=L_{0} \nabla^{2} \mu$ [9, 20], neglecting collision effects. Under the quasi-stationary approximation, thus, the concentration profile around a threedimensional spherical droplet can be obtained by solving the Laplace equation $\nabla^{2} \phi=0$ under the Gibbs-Thomson equation, [2,3, 15,22,23] as $\phi=\bar{\phi}+\left(\phi_{b}-\bar{\phi}\right) R / r$, where $\bar{\phi}$ is the far-field concentration and $\phi_{b}$ is the concentration at the droplet boundary. $\phi_{b}$ is given by the following GibbsThomson equation: $\phi_{b}=-\phi_{e}+\left(\sigma / 2 k_{\mathrm{B}} T \phi_{e} \tau\right)(1 / R)$. Note that the this $1 / r$ dependence of $\phi(r)$, or $\mu(r)$, originates from the conserved nature of the order parameter. For $\delta \phi(=\phi-\bar{\phi})$, thus, we have the following relations:

$$
\delta \phi(r)=\delta \phi_{b} \frac{R}{r}(r>R), \quad \delta \phi(r)=\delta \phi_{b}(r<R),
$$

where $\delta \phi_{b}=\phi_{b}-\bar{\phi}$.

This diffuse concentration change around droplets, $\delta \phi(r)$, qualitatively alters the preceding conclusion that there are no velocity fields for droplet phase separation except for thermal noises. In the preceding discussion, the relation $\nabla \phi \cong\left(\partial \phi_{\text {int }} / \partial \zeta\right) \vec{n}$ are used. This relation is reasonable as the first approximation and can be used for the case of bicontinuous phase separation, where the energy associated with a sharp interface governs the fluid dynamics. For the case of droplet phase separation, $\vec{F}_{\phi}^{i n t}$ given by Eq. (6) does not produce any velocity fields, as described before; and, thus, the first-order corrections do play important roles. Accordingly, we should use the following relation that include the first-order term of $\delta \phi$, instead of Eq. (7):

$$
\nabla \phi \cong\left(\frac{\partial \phi_{i n t}}{\partial \zeta}\right) \vec{n}+\nabla \delta \phi
$$

Here we assume that the phase having a positive value of $\phi$ is the minority phase. In such a case, we have the relation $R_{1}=R_{2}=-R$ for a spherical droplet. Equation (9) should be modified using Eq. (11) to include the additional thermodynamic force

$$
\begin{aligned}
\frac{\vec{F}_{\text {grad }}}{k_{\mathrm{B}} T} & =-K\left[\frac{\partial \phi_{i n t}}{\partial \zeta} \vec{\nabla} \cdot \vec{n}+\nabla^{2} \delta \phi\right] \nabla \delta \phi \\
& \cong \frac{K}{R}\left[4 \phi_{e}+\delta \phi_{b}\right] \delta(\zeta) \nabla \delta \phi
\end{aligned}
$$


Here we use $\partial \phi_{\text {int }} / \partial \zeta \cong 2 \phi_{e} \delta(\zeta)$. We neglect the second term since it is smaller than the first term by a factor of $\sim \xi / R$. This interface force produces the following new velocity fields for droplet phase separation in addition to the thermal velocity noises $\vec{\imath}$ :

$$
\vec{v}_{\text {grad }}(\vec{r})=\int d \overrightarrow{r^{\prime}} \boldsymbol{T}\left(\vec{r}-\overrightarrow{r^{\prime}}\right) \cdot \vec{F}_{\text {grad }}\left(\overrightarrow{r^{\prime}}\right) .
$$

This is the theoretical basis of fluid motion induced by diffuse concentration change around droplets.

Next we estimate the interdroplet interaction via $\vec{F}_{\text {grad }}$. First we consider the coupling between a single spherical particle with a radius of $R$ at the origin and the concentration field of a constant gradient given by $\vec{E} \cdot \vec{r}$. This gradient induces the anisotropic components in the force given by Eq. (12). Using the analogy between this problem and the change of electric potential caused by a metallic sphere in an electric field [15],22], we can estimate the resulting matrix concentration profile around the spherical droplet, $\delta \phi_{E}$, as

$$
\delta \phi_{E}(\vec{r})=\delta \phi(\vec{r})+\vec{E} \cdot \vec{r}-\vec{E} \cdot \vec{r} R^{3} / r^{3} .
$$

Using Eq. (14), we obtain the total force acting on the droplet by integrating the terms containing $E$ in Eq. (12) as

$$
\begin{aligned}
\frac{\vec{F}_{d r o p}}{k_{\mathrm{B}} T} & =\frac{4 K \phi_{e}}{R} \int d \vec{r} \delta(|\vec{r}|-R) \nabla \delta \phi_{E}(\vec{r}) \\
& =16 \pi K R \phi_{e} \vec{E} .
\end{aligned}
$$

Using this relation, we can straightforwardly calculate the total force on a droplet at $\overrightarrow{r_{1}}$ due to the concentration fields of a neighboring droplet at $\overrightarrow{r_{2}}$ by simply replacing the concentration gradient of $\vec{E}$ by $\nabla \delta \phi(\vec{r})\left(\vec{r}=\overrightarrow{r_{1}}-\overrightarrow{r_{2}}\right)$ as

$$
\vec{F}_{R}=-16 \pi k_{B} T K R^{2} \phi_{e} \delta \phi_{b} \frac{\vec{r}}{r^{3}} .
$$

Here we assume the radii of both droplets are $R$. This force can be easily estimated for the case of $\bar{\phi}=-\phi_{e}$ and it gives the strongest force [24]: $\vec{F}_{R}^{\max }=-\gamma k_{B} T R \frac{\vec{r}}{r^{3}}$, where $\gamma=16 \pi \sigma_{0}=5 \sim 10$. Here, we use $\delta \phi_{b}=$ $(2 / 3) \phi_{e}(\xi / R), \quad \xi^{2}=K / 2 \tau$, and $\sigma=4 K \phi_{e}^{2} / 3 \xi \sim$ $\sigma_{0} k_{\mathrm{B}} T / \xi^{2}\left(\sigma_{0}=0.1 \sim 0.2\right.$ is the universal constant). Here it should be noted that this force is not due to the interaction between two droplets in the exact sense, but due to the coupling between a droplet interface and the concentration gradient created by the neighboring droplet.

For a fluid system, the resulting droplet velocity $\vec{v}_{R}^{\max }$ can be estimated by balancing the frictional force $\vec{F}_{S}=$ $-5 \pi \eta R \vec{v}_{R}$ and $\vec{F}_{R}^{\max }$, as

$$
\vec{v}_{R}^{\max } \sim \frac{\vec{F}_{R}^{\max }}{5 \pi \eta R}=\frac{\vec{F}_{R}^{\max } D_{R}}{k_{\mathrm{B}} T}=-\frac{\gamma D_{R} R \vec{r}}{r^{3}} .
$$

Here $D_{R}$ is the diffusion constant of a droplet of radius $R$ and $D_{R}=k_{\mathrm{B}} T /(5 \pi \eta R)$. It is worth noting that a simple dimensional analysis of Eq. (17) tells us that this new mechanism alone leads to the domain growth law of $R \sim t^{1 / 3}$.

On the basis of the above results, we here estimate the maximum coarsening rate including both Browniancoagulation mechanism (thermal noise effects) and the above diffusion-coupling mechanism (see, on the calculation technique, Refs. 5, 15,25). Let us consider a droplet fixed at the origin in the uniform cloud containing $n$ droplets per volume. We suppose the coalescence process is much quicker than the collision interval, which is a reasonable approximation for a fluid system [5]. The boundary conditions for $n$ are (i) $n=0$ at $r=R, t>0$, and (ii) $n=n$ as $r \rightarrow \infty$. The flow of droplets to the origin $I$ is given by $I(r)=4 \pi r^{2} j(r)$ with $j(r)=-D_{R} \partial n / \partial r+n v_{R}$. Note that $I(r)$ should be constant with $r$. Using $I(r)=I$ and the above boundary conditions, we finally get

$$
\frac{d n}{d t}=2 n I=-\frac{8 \pi n^{2} D_{R} R \gamma}{1-\exp (-\gamma / 2)}
$$

where the factor 2 accounts for the fact that the droplet at the origin is free to move. Using the relation $n=$ $\Phi_{d} /\left(\frac{4 \pi}{3} R^{3}\right)$, we obtain from Eq. (18) the following domain growth law:

$$
R=\left[6 \gamma \Phi_{d}\left(D_{R} R\right)\right]^{1 / 3} t^{1 / 3}
$$

The corresponding scaled coarsening law is given by

$$
(R / \xi)^{3}=6 \gamma \Phi_{d} \tau
$$

where $\tau=t / \tau_{\xi}\left[\tau_{\xi}=\xi^{2} / D_{\xi}\left(D_{\xi}=k_{B} T / 5 \pi \eta \xi\right)\right]$.

The time exponent of this final relation is the same as Brownian-coagulation mechanism, but the prefactor is larger by a factor of $\gamma$ than it. This result is quantitatively consistent with the recent careful experimental studies on droplet phase separation in space by Perrot et al. 26]: They demonstrated the $t^{1 / 3}$ law with the twice larger prefactor than that of Brownian-coagulation mechanism [see Eq. (1)], using the relation $k_{d}=12 \Phi_{d}$ [5]. This means $\gamma^{1 / 3} \sim 2.6$, if we use the relation $k_{d}=6 \Phi_{d}$, which we believe is the correct one [6]. In our estimation $\gamma^{1 / 3}=1.7 \sim 2.2$ at maximum, and the agreement is satisfactory at least on a qualitative level. If we take into account the deviation of $\bar{\phi}$ from $\phi_{e}$ [24], $\gamma^{1 / 3}$ becomes smaller to be between 1 and 2 . We need a more analytical approach for the more accurate estimation of $\gamma$.

Finally, we consider novel hydrodynamic effects unique to fluid systems that are not included in the above calculation: (i) Direct hydrodynamic interactions: Interdroplet collision and the resulting shape relaxation (or the relaxation of curvature) directly induces hydrodynamic flow via Eq. (5) and leads directly to another 
collision. This effect likely leads to $v \sim \sigma / \eta$ and $R \sim t$ as first demonstrated by Nikolayev et al. 16 and also by us 14. This mechanism is likely important only for the case of a very high droplet density 14,16. Since this problem is beyond the scope of this paper focusing on the growth law of $R \sim t^{1 / 3}$, we do not discuss it in detail here. (ii) Coupling between hydrodynamic and diffusion modes: The quick hydrodynamic coalescence after a collision could leave the droplet out of local equilibrium and temporally cause a strong diffusion field around it. In our previous papers [27], we discuss the effects of interface quench induced by the quick hydrodynamic reduction of the interfacial energy, focusing on bicontinuous phase separation. We expect a similar, but qualitatively different, dynamic effect for the process of a quick hydrodynamic coalescence after interdroplet collision for droplet phase separation 12,13]: The concentration profile around a droplet just after the collision is about the same as that before the collision since the diffusion process is retarded from the hydrodynamic one. Note that only diffusion can change the concentration profile. Further, the radius of the droplet increases from $R$ to $R^{\prime}$ immediately by collision, satisfying the volume conservation. (a) This increases the interaction strength by a factor of $\left(R^{\prime} / R\right)$ for a droplet experiencing collision and increases the probability of the subsequent collisions. This situation can be realized since the characteristic time of droplet fusion $t_{\text {fusion }} \sim \eta R / \sigma$ is generally much shorter than the characteristic material diffusion time for a droplet $t_{\text {diff }} \sim R^{2} / D_{\xi}$ : Using the relation $\sigma \sim \sigma_{0} k_{\mathrm{B}} T / \xi^{2}$, we obtain $t_{\text {fusion }} / t_{\text {diff }} \sim \xi / 10 R$. The strong excess diffusion fields induced by collision lasts for about $t_{\text {diff }}$ after the interdroplet collision. (b) More importantly, the collision significantly increase the local value of $\nabla^{2} \delta \phi\left(\right.$ or $\nabla^{2} \mu$ ) itself around the droplet for a period of $t_{\text {diff }}$ and, thus, produce the strong attractive interactions between the droplet experiencing the collision and the surrounding droplets via Eq. (12). This leads to the subsequent collisions.

All these effects [(i), (ii)(a), and (ii)(b)] likely strengthen "gradient-induced coupling mechanism" selectively for droplets experiencing collision and lead to the "collision-induced collision" phenomena [12 13 that a droplet experiencing collision has more probability of the subsequent collisions. Such behavior is actually observed experimentally in droplet phase separation 12]. This mechanism can be important when $t_{\text {coll }}<$ $t_{\text {diff } f}$. Here $t_{\text {coll }}$ is the characteristic interval of droplet collision by the hydrodynamic translational motion of droplets. It is estimated as $t_{\text {coll }}(L)=\int_{0}^{L / 2} d r / v_{R}^{\max }=$ $L^{3} /\left(24 D_{R} R \gamma\right)$, where $L$ is the average interdroplet distance. Since $L^{3}=(4 \pi / 3) R^{3} / \Phi_{d}, t_{\text {coll }}=\pi R^{2} / 18 \gamma \Phi_{d} D_{R}$. The ratio $t_{\text {coll }} / t_{\text {diff }}$ is, thus, estimated as $t_{\text {coll }} / t_{\text {diff }} \sim$ $\pi R / 18 \gamma \Phi_{d} \xi \sim 0.02(R / \xi) \Phi_{d}$. Thus, this effect is likely important for the stage where $R<\sim 200 \xi$, for example, at $\Phi_{d} \sim 0.25$. This relation also suggests that in the very late stage the evaporation-condensation mechanism plays an important role. However, there have so far been no reliable experiments on such an extremely late stage.

In summary, we study the coarsening mechanism of droplet phase separation. We find that the interdroplet interaction caused by the anisotropic coupling of concentration fields can accelerate droplet coarsening, in contrast to the conventional understanding that there are no interdroplet interactions, which is the precondition of Brownian-coagulation mechanism. We obtain the domain coarsening law of $R \sim t^{1 / 3}$, whose prefactor is at least larger than that of Brownian-coagulation mechanism. We call this new mechanism of droplet coarsening "gradient-induced coupling mechanism". We also demonstrate the possibility that the strong coupling between velocity and diffusion fields may lead to a new qualitative effect, "collision-induced collision". Further theoretical and experimental studies are highly desirable for the clear underastanding of this problem, including another effect of "collision-induced collision via flow" 14,16.

The author is grateful to T. Ohta, M. Doi, Y. Oono, T. Araki, and T. Kawakatsu for valuable discussions. He also thanks A.E. Kalmykov and V.S. Nikolayev for sending their papers [11,16]. This work was partly supported by a Grant-in-Aid from the Ministry of Education, Science, and Culture, Japan, and also by a grant from Toray Science Foundation.

[1] J.D. Gunton, M. San Miguel, and P. Sahni: in Phase Separation and Critical Phenomena, edited by C. Domb and J.H. Lebowitz, (Academic, London, 1983), Vol. 8.

[2] A.J. Bray, Adv. Phys. 43, 357 (1994).

[3] I.M. Lifshits and V.V. Slyozov: J. Phys. Chem. Solids 19, 35 (1961); C. Wagner: Z. Electrochemie 65, 581 (1961).

[4] K. Binder and D. Stauffer: Adv. Phys. 25, 343 (1976).

[5] E.D. Siggia: Phys. Rev. A 20, 595 (1979).

[6] Siggia derived $k_{d}=12 \Phi_{d}$, but we think the relation $k_{d}=6 \Phi_{d}$ is the right one (see also Ref. [15])

[7] T. Ohta: Ann. Phys. (N.Y.) 158, 31 (1984).

[8] D.A. Huse: Phys. Rev. B 34, 7845 (1986).

[9] G.F. Mazenko: Phys. Rev. E 50, 3485 (1994).

[10] N.C. Wong and C.M. Knobler: Phys. Rev. A 24,3205(1981).

[11] V.I. Aver'yanov and A.E. Kalmykov: Sov. J. Glass Phys. Chem. 18, 288 (1992).

[12] H. Tanaka: Phys. Rev. Lett. 72, 1702 (1994).

[13] H. Tanaka: J. Chem. Phys. 103, 2361 (1995).

[14] H. Tanaka: J. Chem. Phys. 10099 (1996).

[15] V.G. Karpov: Phys. Rev. Lett. 75, 2702 (1995).

[16] V.S. Nikolayev, D. Beysens, and P. Guenoun, Phys. Rev. Lett. 76, 3144 (1996). 
[17] Karpov has recently developped an analytical theory of coarsening of nuclei based on the similar idea as ours, but he has not considered incompressible hydrodynamics.

[18] K. Kawasaki: Prog. Theor. Phys. 57, 826 (1977).

[19] K. Kawasaki and T. Ohta: Physica 118A, 175 (1983).

[20] A. Onuki: Phase-Transition Dynamics (in Japanese) (Iwanami, Tokyo, 1992).

[21] Here we use the mean-field approximation for simplicity.

[22] P.W. Voorhees and R.J. Schaeffer: Acta Metall. 35, 327 (1987).

[23] M. Tokuyama and K. Kawasaki: Physica 123A, 386 (1984).

[24] It should be noted that the mean-field concentration, $\bar{\phi}=-\phi_{e}+\left(\sigma / 2 k_{B} T \phi_{e} \tau\right)(1 / \bar{R})(\bar{R}$ : the average radius of droplets), for example, gives us slower coarsening. In this most realistic case, interactions between droplets are either attractive or repulsive, depending upon the spatial configuration of droplets; and, thus, we have to solve a more complicated many-body problem.

[25] V.G. Levich, Physicochemical Hydrodynamics, (PrenticeHall, Englewood Cliffs, N.J., 1962).

[26] F. Perrot, P. Guenoun, T. Baumberger, D. Beysens, Y. Garrabos, and B. Le Neindre: Phys. Rev. Lett 73, 688 (1994).

[27] H. Tanaka: Phys. Rev. Lett. 72, 3690 (1994); Phys. Rev. E 51, 1313 (1995). 